\title{
Treating ocular surface disease: new agents in development
}

This article was published in the following Dove Press journal:

Clinical Ophthalmology

I3 April 201 I

Number of times this article has been viewed

\author{
Ahmad M Fahmy \\ David R Hardten \\ University of Minnesota, \\ Minneapolis, MN, USA
}

Correspondence: Ahmad Fahmy

Minnesota Eye Consultants,

710 E. 24th Street, Suite I00,

Minneapolis, MN 55404, USA

$\mathrm{Tel}+\mid$ 6I2 8133632

Fax +I 612 8133602

Email amfahmy@mneye.com
Abstract: This paper reviews recent advances and investigation in the treatment of ocular surface pathology. There is significant investment in this area, paralleling the growing demand for more effective alternatives to current treatments. Clinicians are becoming more aware of surface pathology, yet the ability to treat the most common forms of ocular pathology are still limited to the few medications approved by the US Food and Drug Administration. Medicines and devices currently under investigation are very promising. It is absolutely critical to understand the emerging options and think of their role in the treatment paradigm.

Keywords: dysfunctional tear syndrome, anti-inflammatory drugs, treatment

\section{Introduction}

In the last 15 years, there has been significant research and interest in the area of ocular surface disease. The majority of this research has targeted decreasing ocular surface inflammation and promoting the healthy tear complex. The focus is on the underlying pathogenic pathways, and continues to be guided by careful study of the "immune tone" of the lacrimal apparatus tasked with protecting and nourishing the ocular surface. For example, understanding the cytokine and chemokine concentrations in patients without clinical pathology serves as a reference point for further study of patients suffering from ocular surface disease. ${ }^{1}$ The clinical significance of dysfunctional tear syndrome and ocular surface disease is of increasing importance to clinicians, as we continue to uncover pertinent coincident pathology. When compared with patients without dysfunctional tear syndrome, patients suffering from dysfunctional tear syndrome and ocular surface disease are more likely to have systemic disease, including ischemic heart disease, stroke, cardiac arrhythmias, collagen vascular disease (such as rheumatoid arthritis), and depression. ${ }^{2}$ Attention to these potential medical comorbidities is essential. Numerous studies have also demonstrated ocular surface disease and dysfunctional tear syndrome as increasingly impacting on key tasks of daily living, and have advocated that attention be paid to their implications as a public health problem. ${ }^{3}$

The renewed focus on the development of new treatments can be attributed to a better understanding of the etiology of tear complex breakdown and development of dysfunctional tear syndrome. Along with new data driving the interest in this area, the lack of medicines approved by the US Food and Drug Administration specifically for the treatment of dysfunctional tear syndrome drives companies to invest in the development of the next key molecule. Restasis ${ }^{\mathrm{TM}}(0.05 \%$ cyclosporine, Allergan, Irvine, CA) is 
the only medication currently approved by the Food and Drug Administration for the treatment of dysfunctional tear syndrome. New drugs using alternative mechanisms are being examined by several pharmaceutical companies. There is much to gain from the development of this next key medication. This is further driven by the fact that Restasis sales in 2008 yielded $\$ 444$ million. ${ }^{4}$ Most of the material described in this paper is either still in investigation or offlabel application of available devices or medications.

\section{Immunomodulators}

Several pharmaceutical companies are investing in the development of a potentially more effective cyclosporine formulation. This is largely due to reports of the superior efficacy of the $0.1 \%$ concentration when compared with $0.05 \%$, suggesting that cyclosporine could be even more relevant in the treatment of dysfunctional tear syndrome. Restasis $\mathrm{X}^{\mathrm{TM}}$ (cyclosporine $0.1 \%$ ) is under investigation in Phase II trials by Allergan. ${ }^{5}$ Zyclorin $^{\mathrm{TM}}$ (cyclosporine $0.1 \%$ ), is a topical ophthalmic immunomodulator and immunosuppressive agent under investigation which is building on the success of Restasis. Zyclorin differs from Restasis in that it contains double the concentration of cyclosporine. It is currently under Phase III clinical investigation by Alcon and Sirion Pharmaceuticals. $^{6}$ Cyclokat $^{\mathrm{TM}}$ (cyclosporine $0.1 \%$ ) uses a mechanism of immunomodulation similar to that of Restasis and Zyclorin, and has also completed Phase III investigation. The key distinguishing feature of Cyclokat is the use of a proprietary cationic ophthalmic emulsion as a carrier. Due to the electrostatic attraction between the positively charged eye drop and the negatively charged ocular surface, Cationorm ${ }^{\circledR}$ claims to spread in a more optimal manner over the ocular surface with greater uniformity. ${ }^{7}$

LX-214 (voclosporin) belongs to a class of agents known as calcineurin phosphate inhibitors. Two calcineurin phosphate inhibitors are commercially available in the US, ie, tacrolimus (Protopic ${ }^{\mathrm{TM}}$, Astellas, Tokyo, Japan), which is available as $0.03 \%$ and $0.1 \%$ ointments, and topical pimecrolimus (Elidel ${ }^{\circledR}$, Novartis, Basel, Switzerland), which is available as a $1 \%$ cream. Both tacrolimus and pimecrolimus act on $\mathrm{T}$ lymphocytes, which play a critical role in the inflammatory cascade. The calcineurin phosphate inhibitors are complex compounds that bind to macrophilin-12, an intracellular protein. This complex inhibits the phosphorylase enzyme, calcineurin, which prevents translocation of the nuclear factor of activated T cells. The signaling pathway for nuclear factor of activated $\mathrm{T}$ cells targets specific genes in $\mathrm{T}$ cells, including interleukin (IL)-2, IL-3, IL-4, IL-10, interferon- $\gamma$, and granulocyte-macrophage colony-stimulating factor. Systemic administration of tacrolimus (Prograf ${ }^{\text {TM }}$, Astellas) and cyclosporine is implicated in the development of lymphoma, particularly post-transplant lymphoproliferative disorder and nonmelanoma skin cancers. ${ }^{8}$ The basis of this increased risk for malignancy is related to sustained profound systemic immunosuppression. The lymphomas that occur in this setting are usually B cell lymphomas, which develop weeks to months after therapy. Theoretical concerns that topical administration of calcineurin inhibitors could result in either lymphomas similar to post-transplant lymphoproliferative disorder or nonmelanoma skin cancers are based on the assumptions that enough systemic absorption could occur with the topical use of calcineurin phosphate inhibitors to promote the development of these malignancies. Calcineurin phosphate inhibitors may inhibit DNA repair in epidermal keratinocytes, and prolonged use over many years could result in an increased risk of skin cancer. ${ }^{8}$ However, it is important to emphasize that knowledge of systemic calcineurin phosphate inhibitor-associated malignancies preceded the development and licensing of both tacrolimus and pimecrolimus in topical forms. Their approval as topical medications relied on large clinical trials demonstrating low to negligible blood levels, minimal to no evidence of systemic or cutaneous infections with topical use, and other safety parameters.

Lux Biosciences has reported LX-214 to be very well tolerated in a Phase I study, and there was no difference in tolerability between the vehicle control and the two concentrations tested, ie, $0.2 \%$ and $0.02 \% .{ }^{9}$ These positive early tolerability results with the $0.2 \%$ formulation appear to have an advantage over the formulations discussed earlier.

Tasocitinib (CP-690, 550, Pfizer, New York, NY) is a drug which inhibits the Janus kinase (JAK)3 enzyme, found only in immune cells, and discovered in the course of examining severe combined immunodeficiency disease (also known as "bubble boy disease"). This condition results when mutations occur in the genome responsible for creating the JAK3 enzyme. Because cytokines play pivotal roles in immunity and inflammation, targeting of cytokines and their receptors represents an effective means of treating conditions with inflammation as their hallmark. ${ }^{10}$ JAKs are a small family of receptor-associated kinases that, together with signal transducers and activators of transcription, provide a rapid signaling pathway for cytokines. Four JAKs have been identified, ie, JAK1, JAK2, JAK3, and tyrosine kinase 2. JAK3 has attracted much attention as an anti-inflammatory drug target because of its restricted expression in hematopoietic tissue and 
because of its specific association with the common gamma chain of the IL-2 receptor, which is shared by the receptors for IL-4, IL-7, IL-9, IL-15, and IL-21. ${ }^{10}$ Pfizer has been very active in this field, with evaluation of JAK3 inhibition in a number of inflammatory diseases, including rheumatoid arthritis, asthma, ulcerative colitis, Crohn's disease, psoriasis, and prevention of transplant rejection. ${ }^{11}$ Results from the first Phase II study of Pfizer's JAK3 inhibitor revealed the best reported results so far for a small molecule tested in rheumatoid arthritis. JAK2 gain-of-function mutations (JAK2V617F) trigger a subset of disorders collectively referred to as myeloproliferative disorders. ${ }^{11}$ Advanced JAK2 inhibitors are also being evaluated in Phase II trials for myelofibrosis, polycythemia vera, and essential thrombocythemia. A Phase II study is currently enrolling 280 patients. $^{12}$

Tacrolimus (FK-506) is a potent immunosuppressive agent under investigation in Phase IIB studies for the treatment of dysfunctional tear syndrome. Like Restasis, it also targets inflammatory mediators. Tacrolimus was discovered in 1984 and is used worldwide in patients receiving organ transplantation. It is also being used in the US as a topical eyelid ointment (Protopic $0.03 \%$ ) for the treatment of atopic dermatitis. Although Protopic has been shown to be very effective as an eyelid ointment, it is not currently approved for ophthalmic use. ${ }^{13}$

\section{Secretagogs}

Mucin is a critical component of the healthy ocular surface. It is a glycoprotein that lubricates the epithelium while anchoring the tear film. Diquafosol tetrasodium $2 \%$ (Prolacria $^{\mathrm{TM}}$ ) is a mucin secretagog which has shown promising early results in the treatment of dysfunctional tear syndrome. ${ }^{14}$ Diquafosol is an internally developed $\mathrm{P} 2 \mathrm{Y}_{2}$ agonist targeting the Gq protein-coupled $\mathrm{P} 2 \mathrm{Y}_{2}$ receptor, a member of the adenosine triphosphate receptor family, which triggers a series of events, resulting in nonglandular secretion of fluid (water transport via chloride channel activation), mucin secretion, and possibly lipid production in the meibomian glands. Purinergic receptors are found on three cell types, ie, goblet cells, ciliated epithelial cells, and Type II alveolar cells. Discovery of diquafosol stemmed from research into cystic fibrosis and chronic obstructive pulmonary diseasein the late 1990s. ${ }^{15}$

Phase III six-month results ofdiquafosol tetrasodium 1\% and $2 \%$ have recently been reported. ${ }^{16}$ In this study, corneal and conjunctival staining in the diquafosol treatment groups was significantly lowered compared with placebo as early as two weeks following initiation of treatment. This benefit was maintained throughout the duration of the study. Of note, one week after cessation of therapy, the beneficial effects of treatment were diminished.

In 2007, Brazzell et al reported the results of the relevant US clinical trials at the annual meeting of the Association for Research in Vision and Ophthalmology. ${ }^{14}$ This was a summary of seven US clinical trials in over 2000 subjects for durations ranging from one day to one year. Four of these were Phase III studies (104, 105, 108, and 109). A variety of objective and subjective endpoints were evaluated in these trials, including fluorescein staining of the cornea, lissamine green staining of the conjunctiva, Schirmer's test, tear breakup time, and subject-reported symptoms. Corneal fluorescein staining, total clearing of the ocular surface, and clearing of the central cornea were evaluated. In studies 105 and 109, subjects receiving diquafosol $2 \%$ demonstrated significantly $(P \leq 0.001)$ lower mean corneal and conjunctival staining scores compared with subjects receiving placebo after six weeks of four times daily dosing. In study 105, administration of diquafosol resulted in a $75 \%$ increase in Schirmer scores compared with administration of placebo. Diquafosol has been shown in clinical trials to promote clearing of corneal staining.

In study 105, subjects treated with diquafosol were four times more likely to have a corneal staining score of zero following six weeks of treatment than were subjects treated with placebo. In study 108 , subjects treated with diquafosol were three times more likely to have an ocular staining score of zero following four weeks of treatment than were subjects treated with placebo. In both of these studies, the differences in the proportion of patients with total clearing were statistically significant $(P \leq 0.05)$. In studies 105 and 109 , subjects treated with diquafosol were significantly $(P \leq 0.05)$ more likely to have zero staining in the central region of the cornea at their end-of-treatment clinic visit than were subjects treated with placebo. In study 108 , this difference approached significance $(P=0.052)$. Importantly, in studies 104 and 105, a relationship between central corneal clearing status and signs and symptoms was noted. Subjects in these trials who had a clear central cornea following six weeks of treatment had significantly lower $(P \leq 0.05)$ symptom scores than did subjects who did not have a clear central cornea at six weeks. Diquafosol appears to be one of the more promising new medications in ocular surface disease. A diquafosol 3\% formulation (Diquas ${ }^{\circledR}$ ) gained approval in Japan in April 2010. 
IB-MECA (CF101) is an oral treatment which also uses the common mechanistic pathways shared between purine receptor family members. Binding of CF101 to this adenosine receptor agonist initiates downstream signal transduction pathways, which, in turn, downregulate protein kinases, and inhibit production of tumor necrosis factor- $\alpha$. In addition, CF101 inhibits the proliferation of autoreactive $\mathrm{T}$ cells and production of chemokines. ${ }^{17}$ Clinical investigators undertook a multicenter, randomized, double-masked, placebocontrolled, parallel-group study in 68 patients. ${ }^{17}$ Treatment with CF101 resulted in a statistically significant improvement in the mean change from baseline at week twelve for corneal staining, breakup time, and meniscus height. Interestingly, there was also a significant pressure-lowering effect in the treated group and, as such, there is significant interest in this medication for many reasons.

Rebamipide is an amino acid derivative of $2(1 \mathrm{H})-$ quinolinone which also stimulates mucus secretion. Studies have demonstrated that impaired generation of prostaglandins is evident in the gastric and duodenal mucosa of patients with peptic ulcers. ${ }^{18-21}$ Prostaglandin deficiency, in turn, impedes ulcer healing and increases the future risk of relapse. ${ }^{18}$ Rebamipide is a mucoprotective agent widely used in the treatment of gastric ulcers, and stimulates mucus secretion by inducing prostaglandin E2 production. Rebamipide activates the EP-4 gene, downregulates neutrophil production, and scavenges free radicals. ${ }^{19}$ This agent is currently under Phase III investigation by Acucela Pharmaceuticals in partnership with the Otsuka Pharmaceutical Group. Data presented at the Association for Research in Vision and Ophthalmology annual meeting in 2010 reported $1 \%$ and $2 \%$ concentrations of rebamipide to be more effective than placebo in treating dysfunctional tear syndrome.

Ecabet sodium is another medication targeting mucin secretion, and is currently being marketed in Japan as an oral agent for the treatment of gastric ulcers and ulcerative colitis. It is available in Japan as Gastrom ${ }^{\circledR}$. It targets the prostaglandin E2 pathway, inhibiting pepsin formation and increasing blood flow. Ecabet sodium may also downregulate reactive oxygen species on the ocular surface, functioning similar to an anti-inflammatory agent. ${ }^{22}$ ISTA Pharmaceuticals Inc, is currently investigating ecabet sodium in Phase III trials being performed in a novel controlled adverse environment laboratory setting. This environment mimics environmentally-induced changes to the ocular surface. ISTA has reported trends for improvements in blink rate and Ocular Symptom Disease Index score (subjective assessment of patients' most bother some symptom) in
144 patients, with four times daily dosing, versus placebo, for 43 days.

\section{Disaccharide protectants}

Trehaloseis a naturally occurring disaccharide formed by bonding two glucose molecules together, imparting high water retention, and is synthesized by fungi, plants, and invertebrate animals. It is thought to form a gel as it dehydrates, which allows it to "splint" internal components of cells which would otherwise be damaged by desiccation. Trehalose has been found to have antioxidant properties as well as significant resistance to heat or acidic conditions. ${ }^{23}$ Its molecule bonding properties keep glucose in a closed ring form which promotes better stability. It is prevalent in shrimps and insects, where blood sugar is found in this form. Several studies have demonstrated the healing properties of trehalose in the human corneal epithelium. Matusoet al performed an in vitro study with cultured human epithelial cells which effectively showed a reduction in cell death versus sodium hyaluronate, phosphate-buffered solution, and maltose. ${ }^{23}$ Trehalose has also demonstrated superiority over commercially available lubricants for moderate to severe dysfunctional tear syndrome patients in 36 patients during an eight-week crossover study in which sodium fluorescein tear breakup time and Rose Bengal staining were assessed. ${ }^{24}$

\section{Nonsteroidal anti-inflammatory drugs}

Nonsteroidal anti-inflammatory drugs have been used successfully to address the impact of inflammation on the ocular surface. Targeting the inflammation yields improvement in both subjective and objective signs of ocular surface disease. Using nonsteroidal anti-inflammatory drugs in the treatment of dysfunctional tear syndrome and ocular surface diseasehelps address pain, a key symptom which can be challenging to mitigate, especially given that at times it does not coincide with the clinical picture.

Low-dose bromfenac (Remura ${ }^{\mathrm{TM}}$ ) is under Phase II investigation by ISTA, and has demonstrated improvement in lissamine green and ocular surface disease scores in 38 patients over a six-week period..$^{25}$ A Phase III study was initiated in September 2010. Thirty sites in the US are investigating twice-daily dosing at concentrations of $0.03 \%$ and $0.06 \%$ (both lower than Xibrom ${ }^{\mathrm{TM}} 0.09 \%$ ). Nonsteroidal anti-inflammatory drugs have been implicated in corneal melt and decreased corneal sensitivity. ${ }^{26} \mathrm{~A}$ lowdose nonsteroidal anti-inflammatory drug with a good safety 
profile that yields clinical improvement would be a significant addition to the treatment of ocular surface disease. Another significant advantage of this class of agents is that the onset of action is faster than the typical six to eight week period that is sometimes necessary when using topical cyclosporine.

\section{Corticosteroids}

Decreasing inflammation of the ocular surface is a key step en route to restoring conjunctival and corneal epithelium compromised due to dysfunctional tear syndrome and various other inflammatory conditions such as blepharitis, allergy, and autoimmune disease. We often find it necessary to use steroid drops and ointments intermittently in response to acute disease. Typically the side effect profile with respect to cataract formation or steroid-induced glaucoma limits their chronic use.

EGP-437 is a dexamethasone-derived corticosteroid under investigation in a Phase III study by EyeGate Pharmaceuticals. The use of iontophoresis distinguishes this new treatment from other agents in development. EGP-437 is delivered to the ocular surface via an ocular applicator with a central foam reservoir saturated with the drug. This reservoir is placed in the perilimbal conjunctiva. An electrode is placed on the patient's forehead and the ocular applicator is connected to a small, handheld, programmable generator. The generator supplies a negative charge to the ocular applicator and uses the resulting electrorepulsion to push the drug at a high velocity into the eye. This innovative iontophoretic drug delivery system allows the EGP-437 to be maximally absorbed into the ocular surface and overcomes the problem of low bioavailability. Other topical agents have been plagued by this problem. The ability to bypass barriers en route to the site of action is a significant advantage. Current attempts at applying medicines to the ocular surface have been difficult to implement clinically, and have not been efficient. Both the drug and the device are under Phase III investigation. Titrating the level and duration of current application dictates the quantity of drug which ultimately penetrates the ocular surface. According to EyeGate, the iontophoresis process takes only a couple of minutes. If the drug delivery system proves to be effective in the clinic, this could be a welcome new treatment with many applications, because any drug can be loaded into the applicator. This device may be especially useful in treatment of certain retinal conditions.

Lotoprednol etabonate $0.5 \%$ (Lotemax $^{\mathrm{TM}}$ ) has played an effective role in the treatment paradigm of ocular surface disease by controlling inflammation, with a lower tendency to cause spikes in intraocular pressure and to induce cataract formation. ${ }^{27}$ The addition of Lotemax in an ointment form will allow for anti-inflammatory coverage at night before bedtime, which may be better tolerated than currently available topical steroid preparations.

\section{Omega-3 fatty acids}

Studies have demonstrated the anti-inflammatory impact of orally administered essential fatty acids on the ocular surface. ${ }^{28}$ The naturally occurring essential polyunsaturated fatty acids, omega-3 and omega-6, have been shown to have many positive effects on inflammatory diseases, including rheumatoid arthritis and ulcerative colitis. Alpha-linolenic acid has also been shown to improve the ocular surface when applied topically. Alpha-linolenic acid applied topically to dry eyes in mice induced a decrease in corneal fluorescein staining compared with vehicle. ${ }^{29}$ At the cellular level, this study demonstrated an association with a significant decrease in mediators of inflammation, including IL- $1 \alpha$, tumor necrosis factor-alpha (TNF- $\alpha$ ), and conjunctival TNF- $\alpha$. This led to a decrease in dry eye signs, as well as in inflammatory changes in ocular surface disease. This study was a critical demonstration of the potential of topically applied fatty acids, thus bypassing excess caloric intake and possible gastrointestinal adverse effects often associated with oral supplementation. ${ }^{29}$ This was demonstrated via the controlled adverse environment model to induce dysfunctional tear syndrome.

Upon ingestion, omega 3-molecules, ie, eicosapentaenoic acid and docosahexaenoic acid, are converted to resolvins. Inflammatory molecules, including prostaglandins, leukotrienes, and lipoxins, are all lipid mediators enhancing inflammation. Resolvins were first isolated in exudates formed in the resolution phase of acute inflammation or in tissues, such as the brain, in response to an ischemic insult. They were then shown to be produced by human cells and were identified in human plasma. Following the breakthrough of their identification and full structural composition, they were produced by full organic chemical synthesis to explore their pharmacological properties in various prophylactic and therapeutic disease models. ${ }^{30}$ Experimental evidence to date indicates a significant potential for eicosapentaenoic acidderived resolvin E1 in managing both acute and chronic inflammation, with particularly strong evidence in models of colitis, periodontitis, and arthritis, and including profound disease-modifying properties. In other experimental conditions, the administration of the docosahexaenoicacid analog, NPD1, prevented ischemia-induced brain damage, prevented stress-induced apoptosis of retinal epithelial 
cells, and accelerated the recovery of corneal epithelial lesions. The highly specific stereochemistry of resolvin E1 is required for its biological activity down to picomolar concentrations. Resolvin E1 is eventually deactivated in tissues by oxidation to 18 -oxo-RvE1, and is catabolized following omegahydroxylation. This drug protects the ocular surface and most importantly, promotes healing after the insult has passed. Resolvin RX-10045 (Resolvyx ${ }^{\circledR}$ ) has completed Phase I/II investigation in 232 patients, and demonstrated clinically significant improvement in signs and symptoms of dysfunctional tear syndrome, including dryness, stinging, burning, grittiness, and discomfort. ${ }^{31}$ Symptom relief was reported during the first week of treatment, and lasted throughout the treatment phase of 28 days. Resolvyx will be going forward for Phase III investigation.

\section{Anti-infective agents}

Azithromycin 1\% (AzaSite $\left.{ }^{\mathrm{TM}}\right)$ is an antibiotic which has demonstrated excellent results in the treatment of anterior and posterior blepharitis. ${ }^{32}$ Used synergistically with other treatments promoting aqueous secretion, it has been recognized as a leading medication in combating ocular surface disease. It has been shown to change the consistency of the meibum, loosening debris which causes the plugging of glands critical in the preservation of the healthy tear film. ${ }^{32}$ Inspire Pharmaceutical Inc, is currently pursuing Phase III clinical investigation of AzaSite in the treatment of posterior blepharitis. It is currently approved for the treatment of bacterial conjunctivitis.

\section{Autologous serum}

In addition to secretagogs and molecules targeting the inflammatory cascade, another very important and useful therapy in combating ocular surface disease and dysfunctional tear syndrome has been the application of autologous serum. Our attempts at mimicking the human tear film with synthetic lubricants have been useful, yet lack crucial tear film components, such as epidermal growth factor, neurotrophic growth factor, fibronectin, vitamin A, and lysozymes, all of which play an important role in the maintenance of the ocular surface. Autologous serum has been proven to be beneficial in the treatment of persistent epithelial defects, superior limbic keratoconjunctivitis, dysfunctional tear syndrome, Sjögren's syndrome, and neurotrophic keratopathy. ${ }^{33-36}$ Autologous serum has been available for many years, yet is it not widely used due to the formulation process necessitating a compounding pharmacy, and frequent blood draws from the patient. Patients beginning treatment with autologous serum must be educated regarding the level of commitment necessary for the cost of autologous serum. We feel it is an advanced treatment which is indicated in difficult cases of ocular surface disease and dysfunctional tear syndrome. In addition to reported success in these patients, Kojima et al reported statistically significant superiority of autologous serum to nonpreserved artificial tears in a prospective randomized, case-controlled study looking at 37 eyes of patients suffering from severe dysfunctional tear syndrome. ${ }^{37}$ The autologous serum arm showed superior results in film breakup time, sodium fluorescein and Rose Bengal staining, and pain symptom scores. ${ }^{33} \mathrm{We}$ consider this study to be especially important in evaluating the impact of autologous serum because punctal occlusion was not used as in previous studies, and all patients underwent a two-week washout period prior to treatment.

In addition to topically applied medicines, several novel devices have been applied, with promising results in diagnosing, as well as treating, ocular surface disease and dysfunctional tear syndrome.

\section{Intense pulsed light therapy}

Intense pulsed light therapy has been successfully applied in the treatment of ocular surface disease by targeting meibomian gland dysfunction..$^{38}$ The impact of meibomian gland dysfunction has been increasingly implicated as having a significant role in the breakdown of the healthy tear complex. Meibum secretion serves to preserve healthy tear film. Meibomian gland dysfunction causes the meibum to accumulate deep in the gland, resulting in premature tear evaporation and accumulation of inflammatory mediators onto the ocular surface. Inflammation on the eyelid is evident, as seen by the formation of telangiectatic vessels. These abnormal vessels are also seen in facial rosacea. Telangiectasia formation is the hallmark sign of facial rosacea. Patients receiving intense pulsed light treatment for facial rosacea reported improved ocular surface disease symptoms. This is how early investigation into the use of intense pulsed light as a treatment for meibomian gland dysfunction was born, and a research grant in 2004 allowed Toyos et al to demonstrate the efficacy of intense pulsed light in ocular surface disease. ${ }^{38}$

Intense pulsed light emits energy in the visible spectrum between the near and mid-infrared wavelengths. Hemoglobin absorbs the energy and coagulates, causing thrombosis of the abnormal vessels. ${ }^{39}$ These closed vessels can no longer continue to send proinflammatory mediators to the mebomian gland. We have treated many patients with intense pulsed light and have seen significantly improved meibomian gland func- 
tion and lipid secretion in intense pulsed light-treated patients who had significant meibomian gland dysfunction. When appropriately applied, coupled with topical treatment, intense pulsed light has been shown to be very effective. We predict that this is a treatment that will be widely used.

\section{Amniotic membrane transplantation}

The amniotic membrane is the inner most layer of the placenta, consisting of a thick basement membrane and a vascular stromal matrix..$^{40}$ It has effectively been used as a temporary patch to promote healing of the ocular surface by reducing inflammation and scar formation. ${ }^{40}$ This healing effect lends its use in multiple cases of ocular surface pathology, including corneal ulcers, severe ocular surface desiccation from chemical and thermal burns, limbal stem cell deficiency, and scleral melt. ${ }^{41-43}$ Amniotic membrane transplantation has also been successfully used recently in the treatment of Stevens-Johnson syndrome and toxic epidermal necrolysis. ${ }^{44}$ Recent studies and applications of amniotic membrane provide confirmation of earlier investigations and there is mounting agreement that early intervention with amniotic transplantation in cases of severe pathology is key to preserving the ocular surface and ameliorating visual function.

\section{Conclusion}

Ocular surface disease continues to be a common and important clinical condition. Even though a number of current therapies can improve symptoms and findings in many cases, affected people often live with significant symptoms and disability due to the condition. Much active investigation is being directed towards understanding and treating ocular surface disease more effectively.

\section{Disclosure}

Both authors have no financial interest in the development of any of the products or medications discussed in this article.

\section{References}

1. Carreño E, Enríquez-de-Salamanca A, Tesón M, et al. Cytokine and chemokine levels in tears from healthy subjects. Acta Ophthalmol. 2010; 88:e250-e258.

2. Wang TJ, Wang IJ, Hu CC, Lin HC. Comorbidities of dry eye disease: A nationwide population-based study. Acta Ophthalmol. August 31, 2010. [Epub ahead of print].

3. Miljanović B, Dana R, Sullivan DA, Schaumberg DA. Impact of dry eye syndrome on vision-related quality of life. Am J Ophthalmol. 2007; 143:409-415.

4. Allergan financial reports. Allergan. 2007. Available at: http:// www.allergan.com/assets/pdf/2009AnnualReport.pdf. Accessed February 20, 2011.
5. Allergan pipeline. Allergan. 2011. Available at: http://www.allergan.com/ research_and_development/pipeline.htm\#. Accessed February 20, 2011.

6. Sirion Therapeutics. 2011. Available at:http://www.ophthalmology summit.com/presentations/Sirion.pdf. Accessed February 20, 2011.

7. Eye Therapy. NovagaliPharma. 2011. Available at: http://www.novagali. com/en/eye-therapy/severe-dry-eye/. Accessed February 20, 2011.

8. Ormerod AD. Topical tacrolimus and pimecrolimus and the risk of cancer: How much cause for concern? Br J Dermatol. 2005;153: 701-705.

9. Products and technologies. Lux BioSciences. 2011. Available at: http:// www.luxbio.com/LX214.htm. Accessed February 20, 2011.

10. West K. CP-690550, a JAK3 inhibitor as an immunosuppressant for the treatment of rheumatoid arthritis, transplant rejection, psoriasis and other immune-mediated disorders. Curr Opin Investig Drugs. 2009;10: 491-504.

11. Levine R, Pardanani A, Tefferi A, Gilian G. Role of JAK2 in the pathogenesis and therapy of myeloproliferative disorders. Nat Rev Cancer. 2007;7:673-683.

12. US National Institutes of Health Clinical Trials. 2011. Available at:http://clinicaltrials.gov/ct2/show/NCT00483756. Accessed February 20, 2011.

13. Wyrsch S, Thiel MA, Becht CN. Safety of treatment with tacrolimus ointment for anterior segment inflammatory diseases. Klin Monbl Augenheilkd. 2009;226:234-236.

14. Brazzell R, Kellerman DJ, Schaberg A, Yerxa B, Durham T. Effect of diquafosol on ocular surface health in dry eye disease. Poster presented at the 2007 Annual meeting of the Association for Research in Vision and Ophthalmology. May 6-10, 2007; Fort Lauderdale, FL.

15. Evans R, Johnson F, Kellerman D, et al. Aerosolized INS365 for COPD: Enhanced deposition and clearance using the AERx ${ }^{\circledR}$ delivery system. Poster presented at the American Thoracic Society International Conference. May 5-10, 2000; Toronto, ON.

16. Tauber J, Davitt WF, Bokosky JE, et al. Double-masked, placebocontrolled safety and efficacy trial of diquafosol tetrasodium (INS365) ophthalmic solution for the treatment of dry eye. Cornea. 2004;23: 784-792.

17. Avni I, Garzozi HJ, Barequet IS, et al. Treatment of dry eye syndrome with orally administered CF101: Data from a phase 2 clinical trial. Ophthalmology. 2010;117:1287-1293.

18. Arakawa T, Watanabi T, Fukuda T, Yamasaki K, Kobayashi K. Rebamipide, novel prostaglandin-inducer, accelerates healing and reduces relapse of acetic acid-induced rat gastric ulcer. Comparison with cimetidine. Dig Dis Sci. 1995;40:2469-2472.

19. Matsuda T, Ohno S, Hirohata S, et al. Efficacy of rebamipide as adjunctive therapy in the treatment of recurrent oral aphthous ulcers in patients with Behçet's disease: Arandomised, double-blind, placebocontrolled study. Drugs R D. 2003;4:19-28.

20. Ishiharaa T, Tanakaa K, Tashiroa S, Yoshidaa K, Mizushim T. Protective effect of rebamipide against celecoxib-induced gastric mucosal cell apoptosis. Biochem Pharmacol. 2010;79:1622-1633.

21. Suetsugu H, Ishihara S, Moriyama N, et al. Effect of rebamipide on prostaglandin EP4 receptor gene expression in rat gastric mucosa. $J$ Lab Clin Med. 2000;136:50-57.

22. Munakata W, Liu Q, Shimoyama T, Sawaya M, Umeda T, Sugawara K. Ecabet sodium attenuates reactive oxygen species produced by neutrophils after priming with bacterial lipopolysaccharides. Luminescence. 2003;18:330-333.

23. Matsuo T, Tsuchida Y, Morimoto N. Trehalose eye drops in the treatment of dry eye syndrome. Ophthalmology. 2002;109: 2024-2029.

24. Matsuo T. Trehaloseversus hyaluronan or cellulose in eyedrops for the treatment of dry eye. Jpn J Ophthalmol. 2004;48:321-327.

25. Chandler S, James A, Rowen S, Sher N. Alleviation of dry eye disease symptoms with bromfenacophthalmic solution. Poster presented at the Sixth International Conference on the Tear Film and Ocular Surface Basic Science and Clinical Relevance. September 23-25, 2010: Florence, Italy. 
26. Congdon N, Schein O, Kulajta P, Lubomski L, Gilbert D, Katz J. Corneal complications associated with topical ophthalmic use of nonsteroidal anti-inflammatory drugs. J Cataract Refract Surg. 2001;27:622-631.

27. Pavesio CE, Decory HH. Treatment of ocular inflammatory conditions with loteprednol etabonate. Br J Ophthalmol. 2008;92:455-459.

28. Barabino S, Rolando M, Camicione P, et al. Systemic linoleic and gamma-linolenic acid therapy in dry eye syndrome with an inflammatory component. Cornea. 2003;22:97-101.

29. Rashid S, Jin Y, Ecoiffier T, Barabino S, Schaumberg D, Dana R. Topical omega-3 and omega-6 fatty acids for treatment of dry eye. Arch Ophthalmol. 2008;126:219-225.

30. Serhan C, Krishnamoorthy S, Recchiuti A, Chiang N. Novel antiinflammatory - pro-resolving mediators and their receptors. Curr Top Med Chem. January 25, 2011. [Epub ahead of print].

31. US National Institutes of Health Clinical Trials. 2011. Available at: http://clinicaltrials.gov/ct2/show/NCT00799552. Accessed February 20, 2011.

32. Zhou N, Ma P, Li D-Q, Pflugfelder SC. Azithromycin suppresses pro-inflammatory mediatorsinduced by TLR2 ligand zymosan in human corneal epithelial cells. Poster presented at the Annual meeting of the Association for Research in Vision and Ophthalmology. May 3-7, 2009; Fort Lauderdale, FL.

33. Tsubota K, Goto E, Shimmura S, Shimazaki J. Treatment of persistent epithelial corneal defect by autologous serum application. Ophthalmology. 1999;106:1984-1989.

34. Tsubota K, Goto E, Fujita H, et al. Treatment of dry eye by autologous serum in Sjogren's syndrome. Br J Ophthalmol. 1999;83:390-395.

35. Goto E, Shimmura S, Shimazaki J, Tsubota K. Treatment of superior limbic keratoconjunctivitis by application of autologous serum. Cornea. 2001;20:807-810.
36. Matsumoto Y, Dogru M, Goto E, et al. Autologous serum application in the treatment of neurotrophickeratopathy. Ophthalmology. 2004;111: 1115-1120.

37. Kojima T, Ishida R, Dogru M, et al. The effect of autologous serum eyedrops in the treatment of severe dry eye disease: A prospective randomized case-control study. Am J Ophthalmol. 2005;139: $242-246$.

38. Toyos R. Intense pulsed light for dry eye syndrome. Cataract and Refractive Surgery Today. April 2009;1-3. Available at: http://bmctoday.net/ crstoday/2009/04/article.asp?f=CRST0409_14.php. Accessed February 22,2011 .

39. Mark K, Sparacio R, Voigt A, Marenus K, Sarnoff D. Objective and quantitative improvement of rosacea-associated erythema after intense pulsed light treatment. Dermatol Surg. 2003;29:600-604

40. Tseng S. Amniotic membrane transplantation for ocular surface reconstruction. Biosci Rep. 2001;21:481-489.

41. Lee S, Tseng S. Amniotic membrane transplantation for persistent epithelial defects with ulceration. Am J Ophthalmol. 1997;123: 303-312.

42. Anderson DF, Ellies P, Pires R, Tseng S. Amniotic membrane transplantation for partial stem cell deficiency: Long-term outcomes. Br J Ophthalmol. 2001;85:567-575.

43. Meller D, Pires R, Mack R, et al. Amniotic membrane transplantation for acute chemical or thermal burns. Ophthalmology. 2000;107:980-990.

44. Shay E, Khierkhan A, Liang L, et al. Amniotic membrane transplantation as a new therapy for acute ocular manifestations of Stevens-Johnson syndrome and toxic epidermal necrolysis. Surv Ophthalmol. 2009; 54:686-696
Clinical Ophthalmology

\section{Publish your work in this journal}

Clinical Ophthalmology is an international, peer-reviewed journal covering all subspecialties within ophthalmology. Key topics include: Optometry; Visual science; Pharmacology and drug therapy in eye diseases; Basic Sciences; Primary and Secondary eye care; Patient Safety and Quality of Care Improvements. This journal is indexed on

\section{Dovepress}

PubMed Central and CAS, and is the official journal of The Society of Clinical Ophthalmology (SCO). The manuscript management system is completely online and includes a very quick and fair peer-review system, which is all easy to use. Visit http://www.dovepress.com/ testimonials.php to read real quotes from published authors. 